\title{
$\gamma$-Ray Doppler Factors for Fermi Blazars
}

\author{
J. H. Fan ${ }^{1,2}$, J. H. Yang ${ }^{3}$, Q. F. Zhang ${ }^{4}$, Y. Li ${ }^{4}$, Y. Liu ${ }^{1,2}$ \\ ${ }^{1}$ Center for Astrophysics, Guangzhou University, Guangzhou 510006, China \\ email: jhfan_cn@yahoo.com.cn \\ ${ }^{2}$ Astronomy Science and Technology Research Laboratory of Department of Education of \\ Guangdong Province, Guangzhou 510006, China \\ ${ }^{3}$ Department of Physics and Electronics Science, Hunan University of Arts and Science, \\ Changde 415000, China \\ ${ }^{4}$ Department of Computer Science, Jinan University, Guangzhou 510632, China
}

\begin{abstract}
In this paper, we compiled the X-ray observations for 138 Fermi blazars and calculated their Doppler factors, $\delta_{\gamma}$. The calculated Doppler factors, $\delta_{\gamma}$, are found strongly correlated with the $\gamma$-ray luminosity.
\end{abstract}

Keywords. Galaxies:active-galaxies:BL Lacertae objects-galaxies:quasars-galaxies:jets-Fermi (LAT)

\section{Introduction}

Blazars are an extreme subclass of active galactic nuclei(AGNs), and show rapid and large variability, high and variable polarization, superluminal motions in their radio components, and strong $\gamma$-ray emissions, etc (Abdo et al. 2010; Nolan et al. 2012; Fan 2012).

Since the launch of the new generation of $\gamma$-ray detector, Fermi has detected a lot of blazars (Abdo et al.2010; Nolan et al. 2012). The relativistic beaming effect has been discussed for the Fermi blazars (see Savolainen et al. 2010, and references therein). But the $\gamma$-ray Doppler factors have not been estimated for those Fermi blazars. It is known that the Doppler factors are important in the discussion of blazars, therefore, we will estimate the factors for the Fermi detected blazars with available X-ray emission data. We adopt $\mathrm{H}_{0}=73 \mathrm{~km} \mathrm{~s}^{-1} \mathrm{Mpc}^{-1}$ through this paper.

\section{Doppler Factor Estimation and Conclusion}

The extreme observation properties of blazars, such as rapid variability, high and variable polarization, and superluminal motion can be explained using a relativistic beaming model. The strong $\gamma$-rays detected from blazars imply that the beaming effect is presented in those sources, otherwise, the $\gamma$-rays should have been absorbed due to pair-production on collision with the lower energetic photons. In 1993, Mattox, et al. considered the pairproduction optical depth, they assumed that (1) the X-ray is produced in the same region as the $\gamma$-rays, and that a similar X-ray intensity was extant at the time of the $\gamma$-ray observation, (2) the emission region is spherical, (3) the emission is isotropic, and the size of the emission region is constrained by time variation to be less than $R=c \Delta T /(1+z)$, here $\Delta T$ is the timescale of variability, $\mathrm{c}$ is the speed of light, $\mathrm{z}$ is the redshift. Based on those assumptions and that the optical depth does not exceed unity, one can obtain a lower limit for the $\gamma$-ray Doppler factor, $\delta_{\gamma}$, namely

$$
\delta \geqslant\left[1.54 \times 10^{-3}(1+\mathrm{z})^{4+2 \alpha}\left(\frac{\mathrm{d}_{\mathrm{L}}}{\mathrm{Mpc}}\right)^{2}\left(\frac{\Delta \mathrm{T}}{\mathrm{hr}}\right)^{-1}\left(\frac{\mathrm{F}_{\mathrm{KeV}}}{\mu \mathrm{Jy}}\right)\left(\frac{\mathrm{E}_{\gamma}}{\mathrm{GeV}}\right)^{\alpha}\right]^{\frac{1}{4+2 \alpha}}
$$




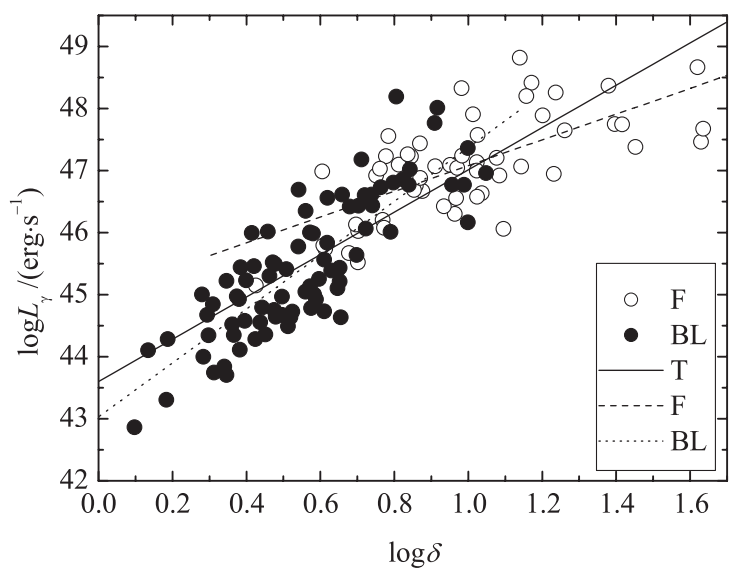

Figure 1. Plot of the $\gamma$-ray luminosity, $\log \nu \mathrm{L}_{\nu}$ (ergs/s) against the $\gamma$-Doppler factor, $\log \delta_{\gamma}$ for the Fermi blazars. The open circles stand for FSRQs, the filled points for BLs. The solid line stands for the best fitting result for the whole sample, the broken line for FSRQs, and the dotted line for BLs.

where $\alpha$ is the $\mathrm{X}$-ray spectral index $\left(\mathrm{F}_{\nu_{\mathrm{X}}} \propto \nu_{\mathrm{X}}^{-\alpha}\right), \mathrm{F}_{\mathrm{KeV}}$ is the flux density at $1 \mathrm{KeV}, \mathrm{E}_{\gamma}$ is the energy at which the $\gamma$-rays are detected, and $\mathrm{d}_{\mathrm{L}}$ is the luminosity distance, which can be expressed in the form $d_{L}=\frac{c}{H_{0}} \int_{1}^{1+z} \frac{1}{\sqrt{\Omega_{M} x^{3}+1-\Omega_{M}}} \mathrm{dx}$ from the $\Lambda-C D M$ model with $\Omega_{\Lambda} \simeq 0.7, \Omega_{M} \simeq 0.3$ and $\Omega_{K} \simeq 0.0$.

Estimation of $\delta_{\gamma}$ When the relation is adopted to 138 Fermi blazars with available Xray data, the $\gamma$-ray Doppler factors, $\delta_{\gamma}$ 's are obtained by assuming that $\Delta \mathrm{T}=24$ hours, the plot of $\delta_{\gamma}$ 's vs the $\gamma$-ray luminosity is shown in Fig. 1.

Discussions and Conclusion From the Fermi mission, we can see that most of the identified detected point sources are blazars (Nolan et al. 2012). So, we can take $\gamma$-ray emissions as one of the observational properties of blazars. From the obtained $\delta_{\gamma}$ 's, and the calculated $\gamma$-ray luminosities(see Fan et al. 2012$)$, we have $\log L_{\gamma}(\mathrm{erg} / \mathrm{s})=(3.41 \pm$ $0.18) \log \delta+(43.60 \pm 0.14)$ for the whole sample, which confirms that the $\gamma$-rays are strongly beamed. However, the slope and constant $\left(\log L_{\gamma}(\mathrm{erg} / \mathrm{s})=(2.07 \pm 0.31) \log \delta+(45.00 \pm\right.$ $0.32))$ for FSRQs are different from those $\left(\log L_{\gamma}(\mathrm{erg} / \mathrm{s})=(4.34 \pm 0.33) \log \delta+(43.03 \pm\right.$ 0.19)) for BL Lacs. Do flat spectrum radio quasars have different $\gamma$-ray origin than BL Lacs? Or they have different dependence on the $\gamma$-ray Doppler factors? or they have different $\Delta T$ ?

\section{Acknowledgements}

The work is partially supported by the National Natural Science Foundation of China (NSFC 11173009), the Bureau of Education of Guangzhou Municipality(No.11 Sui-JiaoKe[2009]), Guangdong Province Universities and Colleges Pearl River Scholar Funded Scheme(GDUPS)(2009), and Yangcheng Scholar Funded Scheme(10A027S).

\section{References}

Abdo, A. A., Ackermann, M., Ajello, M., et al. 2010, ApJS 188, 405

Fan, J. H. 2012, Journal of Guangzhou University(Nat. Sci. Edi), Vol. 11, No. 2, 18

Fan, J. H., Yang, J. H., Zhang, J. Y. et al. 2012, PASJ, (submited)

Nolan, P. L., Abdo, A. A., Ackermann, M., et al. 2012, ApJS, 199, 31 